\title{
Bifidobacteria may be beneficial to intestinal microbiota and reduction of bacterial translocation in mice following ischaemia and reperfusion injury
}

\author{
Honggang Wang, Wei Zhang, Lugen Zuo, Weiming Zhu*, Bin Wang, Qiurong Li and Jieshou Li \\ Department of General Surgery, Jinling Hospital, Medical School of Nanjing University, No. 305 East Zhongshan Road, \\ Nanjing, People's Republic of China
}

(Submitted 5 January 2012 - Final revision received 17 August 2012 - Accepted 19 August 2012 - First published online 5 November 2012)

\begin{abstract}
The aim of the present study was to determine the effect of peroral bifidobacteria on the intestinal microbiota, barrier function and bacterial translocation (BT) in a mouse model of ischaemia and reperfusion (I/R) injury. A total of twenty-four male BALB/c mice were randomly allocated into three groups: (1) sham-operated, (2) I/R and (3) I/R injury and bifidobacteria pretreatment ( $10^{9}$ colony-forming units/d). Bifidobacteria were administered daily intragastrically for 2 weeks before induction of $\mathrm{I} / \mathrm{R}$. Subsequently, samples of caecal content, intestinal mucosa, ileal segments, blood, mesenteric lymph nodes (MLN) and distant organs (liver, spleen and kidney) were prepared for examination. In the I/R model, barrier dysfunction (caecal microbiota dysbiosis, disruption of tight junction (TJ), increased epithelial cell apoptosis, disruption of mucosa and multiple erosions) in the intestine was observed, associated with increased BT to extraintestinal sites. The ratio of BT to MLN and distant organs in mice exposed to I/R injury was $62.5 \%$, which was significantly higher than the sham-operated group. However, pretreatment of animals with bifidobacteria prevented I/R-induced BT, reduced pro-inflammatory cytokine release, the levels of endotoxin, intestinal epithelial cell apoptosis, disruption of TJ and increased the concentration of SCFA, resulting in recovered microbiota and mucosal integrity. Bifidobacteria may be beneficial in reducing BT in I/R injury of mice. Therefore, peroral administration of bifidobacteria is a potential strategy to prevent I/R-induced BT and intestinal barrier dysfunction.
\end{abstract}

Key words: Bifidobacteria: Ischaemia and reperfusion: Bacterial translocation: Microbiota

The human intestine is colonised by trillions of microorganisms, including hundreds of different species of bacteria and viruses ${ }^{(1)}$. These microbes, collectively referred to as the commensal microbiota, have an important role in human nutrition and health, by promoting nutrient supply, preventing pathogen colonisation and shaping and maintaining normal mucosal immunity ${ }^{(2)}$. However, the relationship between indigenous intestinal microbes and their hosts can shift from commensalism towards pathogenicity in certain diseases. Loss of normal microbiota is responsible for overgrowth of opportunistic pathogens that are normally inhibited, which occurs in antibiotic-associated colitis ${ }^{(3)}$. Bacterial translocation (BT) is defined as the passage of viable bacteria from the gastrointestinal tract through the mucosal epithelium to other sites, such as the mesenteric lymph nodes (MLN) and distant organs after gut barrier failure ${ }^{(4)}$. The intestinal epithelial cell is extremely susceptible to ischaemia-reperfusion (I/R) injury, and even short periods of ischaemia can provoke substantial partial tissue damage with the potential for other problems ${ }^{(5)}$. Intestinal $\mathrm{I} / \mathrm{R}$ injury is a serious condition, which may result from severe trauma, septic or haemorrhagic shock, intestinal mesenteric vascular ischaemia, necrotising enterocolitis or certain surgical procedures including small bowel transplantation and abdominal aortic surgery ${ }^{(6-8)}$. It is considered that intestinal $\mathrm{I} / \mathrm{R}$ not only results in the injury of the intestine itself, particularly the small intestine, but may also cause multiple organ dysfunction syndrome owing to damage of the intestinal mucosal barrier ${ }^{(9)}$. Injury to the intestinal mucosal barrier during intestinal I/R leads to translocation of bacteria and endotoxin, with the systemic dissemination linked to the endogenous production of pro-inflammatory cytokines, such as TNF- $\alpha$ and IL- 6 , and to the systemic inflammatory response syndrome ${ }^{(10-12)}$. Consequently, intestinal ischaemia injury and subsequent reperfusion have been proposed as major mechanisms involved in BT occurrence in several clinical conditions.

Probiotics are usually defined as live micro-organism supplements, which when administered in adequate amounts confer a health benefit on the host by improving its intestinal microbial balance ${ }^{(13)}$. It is known that one of the primary mechanisms promoting BT in animal models is the disruption of the ecologic intestinal equilibrium to allow

Abbreviations: BT, bacterial translocation; I/R, ischaemia-reperfusion; MLN, mesenteric lymph nodes; ZO-1, zona occludens-1. 
intestinal bacterial overgrowth ${ }^{(14)}$. The main potentially health-enhancing bacteria are the bifidobacteria and lactobacilli, both of which belong to the lactic acid bacteria group $^{(15)}$. These two genera do not include any significant pathogenic species and their dominance in the faeces of breast-fed babies is thought to impart protection against infection $^{(16)}$. As probiotic agents, bifidobacteria have been studied for their efficacy in the prevention and treatment of a broad spectrum of animal and/or human gastrointestinal infections through production of SCFA, especially acetate $^{(17,18)}$. However, little is known about how bifidobacteria improve the intestinal barrier function and prevent intestinal I/R-induced BT. In the present study, we mainly investigated the effects of peroral bifidobacteria, including Bifidobacterium longum, Bifidobacterium bifidum and Bifidobacterium adolescentis on intestinal microbiota and on BT to MLN and distant organs in a mouse model of $\mathrm{I} / \mathrm{R}$ injury.

\section{Materials and methods}

\section{Animals}

Male BALB/c mice, 6-8 weeks old, weighing 22-25 g, were housed in plastic-bottomed wire-lidded cages and kept at $25^{\circ} \mathrm{C}$ with a daily $12 \mathrm{~h}$ light $-12 \mathrm{~h}$ dark cycle. The mice had free access to water and chow ad libitum. All animals were acclimatised at least $3 \mathrm{~d}$ before use. The experimental procedures were performed in accordance with the Guidelines for Animal Experiments at the Jin Ling Hospital and approved by the Ethical Committee at the Jin Ling Hospital (Jiangsu, China).

\section{Preparation and administration with bacterial cells}

Bifidobacteria (containing B. longum, B. bifidum and $B$. adolescentis) were provided by the Nanjing University (Nanjing, China) as a freeze-dried powder containing $1 \times 10^{10}$ colony-forming units/g. At 2 weeks before the $\mathrm{I} / \mathrm{R}$ experiment, the mice were orogastrically administered $0 \cdot 2 \mathrm{ml}$ normal saline or bifidobacteria $\left(1 \times 10^{9}\right.$ cells in $0.2 \mathrm{ml}$ normal saline) daily and standard chow and water ad libitum. In total, three groups of mice were studied: (1) sham-operated mice (fed with normal saline and underwent laparotomy, without I/R injury); (2) $\mathrm{I} / \mathrm{R}$ mice (fed with normal saline and underwent laparotomy, with intestinal $\mathrm{I} / \mathrm{R}$ injury); (3) bifidobacteria $+\mathrm{I} / \mathrm{R}$ mice (pretreated with bifidobacteria and underwent the $\mathrm{I} / \mathrm{R}$ procedure).

\section{Ischaemia and reperfusion experiment}

Mice were anaesthetised with ketamine $(120 \mathrm{mg} / \mathrm{kg}$, intraperitoneally) and a laparotomy was performed. The intestinal I/R protocol employed has been previously described ${ }^{(19)}$. The superior mesenteric artery was isolated and ischaemia was induced by totally occluding the superior mesenteric artery for $60 \mathrm{~min}$. For measuring BT and barrier dysfunction in mice, reperfusion was re-established. At $3 \mathrm{~h}$ after reperfusion, a laparotomy was performed to determine microbiological analysis of caecal content, BT to MLN and distant organs, levels of plasma endotoxin and pro-inflammatory cytokines, damage of ileal villus and tight junction (TJ).

\section{Microbiological analysis}

The proximal part of the caecum was dissected and the caecal content was extracted using aseptic techniques, weighed, homogenised and serially diluted to obtain $10^{-3}, 10^{-5}$ and $10^{-7}$ concentrations. Aliquots $(100 \mu \mathrm{l})$ of each dilution were plated on the appropriate plates (EC Agar Medium, for Enterococci; Eosin Methylene Blue Agar Medium, for Enterobacteria; Bacteroides Bile Esculin Agar Medium, for Bacteroides fragilis group; MRS Agar Medium, for Lactobacillus and TPY Agar Medium for bifidobacteria, Oxoid Ltd). The cultures were incubated for $24 \mathrm{~h}$ for aerobic bacterial culture and $72 \mathrm{~h}$ for anaerobic culture at $37^{\circ} \mathrm{C}$. The mean colony counts obtained from the agar plates were expressed as colony-forming units/g of content.

\section{Determination of the SCFA in caecum contents}

The analysis of SCFA was carried out by GC as follows. Supernatants from $1 \mathrm{ml}$ of the homogenised caecum content samples were obtained by centrifugation $(10000 \mathrm{~g}$, $\left.30 \mathrm{~min}, 4^{\circ} \mathrm{C}\right)$ and filtration $(0.45 \mu \mathrm{m})$. A chromatographic system composed of a gas chromatograph, HP5890 II (Hewlett Packard), coupled with an ion flame detector and a MS 5973N detector (Agilent Technologies, Inc.) was used for quantification according to Salazar et $a l .^{(20)}$. 4-Methyl valeric acid was used as internal standard.

\section{Bacterial translocation}

Using aseptic techniques, tissue samples from the MLN, liver, spleen and kidney were taken for bacteriological cultures. Collected tissue samples were weighed and $0 \cdot 1 \mathrm{~g}$ of each was homogenised in a tissue grinder with $0.9 \mathrm{ml}$ sterile saline. The homogenates were diluted and $100 \mu$ l dilutions were taken and cultured on MacConkey's agar at $37^{\circ} \mathrm{C}$ for $24 \mathrm{~h}$. Any growth in the plates of bacteria was expressed as colony-forming units/g of tissue. The result of cultures was considered to be positive when more than $10^{2}$ colonies/g of tissue were found.

\section{Detection of plasma endotoxin, TNF- $\alpha$, and IL-6}

Levels of endotoxin in blood were assessed using a spectrophotometric limulus amoebocyte lysate assay, according to the manufacturer's instructions (Beijing Med \& Chem Institute). Endotoxin was expressed as $\mathrm{pg} / \mathrm{ml}$. This method was sensitive to $5 \mathrm{pg}$ endotoxin per $\mathrm{ml}$. Sera were assayed for cytokine levels (TNF- $\alpha$ and IL-6) using a multiplex bead-based immunoassay kit (Bio-Rad). Cytokine assays were performed as described by the manufacturer's protocol. Each reaction in the kit was done in duplicate, with the experiment repeated three times. 


\section{Pathological analysis}

Ileum tissue pieces were formalin-fixed and embedded in paraffin. Thereafter, $4 \mu \mathrm{m}$-thick sections were stained with haematoxylin and eosin. Light microscopic studies were reviewed by a pathologist blinded to the protocol. The degree of intestinal tissue injury was evaluated and each was graded on a scale of $0-5$, identical to that originally described by Chiu $e t \mathrm{al}^{(21)}$. Chiu's score was graded as follows: grade 0 was defined as normal mucosa and grade 1 was the development of a subepithelial space at the tips of the villi. This space was more extended in grade 2 . In grade 3 , there was massive epithelial lifting down the sides of the villi. In grade 4 , the villi were denuded of epithelium and grade 5 was characterised by a loss of the villi themselves.

\section{Transmission electron microscopy of tight junction}

Sections of ileum $(2 \mathrm{~mm})$ were fixed for $2 \mathrm{~h}$ in $4 \%$ buffered glutaraldehyde. The sections were cut into smaller pieces, post-fixed in $1 \%$ osmium tetroxide $\left(\mathrm{OsO}_{4}\right)$, sequentially dehydrated through graded alcohols, infiltrated through Epon812 and then embedded in resin. Thin sections were cut and stained with uranyl acetate and lead citrate, and examined with a Hitachi H-600 transmission electron microscope (Hitachi) operated at $75 \mathrm{kV}$ at a magnification of $20000 \times$. TJ were considered to have increased permeability when the electron-dense marker penetrated into the junctional complex ${ }^{(22)}$.

\section{Western blot analysis of tissue claudin-1, occludin and zona occludens-1 content}

Intestinal tissue samples were homogenised in lysis buffer (20 mm-Tris-HCl (pH 7.5), 1\% Triton X 100, $0.2 \mathrm{M}-\mathrm{NaCl}$, 2 mм-EDTA, 2 mm-ethylene glycol tetraacetic acid (EGTA), 1 $\mathrm{m}$-dithiothreitol (DTT) and $2 \mathrm{~m}$-aprotinin). Proteins $(50 \mu \mathrm{g})$ were electrophoresed using SDS-PAGE (8\%) and transferred to a nitrocellulose membrane. Membranes were blocked with non-fat dried milk in Tris-buffered saline containing $0.05 \%$ Tween- 20 for $1 \mathrm{~h}$ at room temperature and incubated with a rabbit anti-mouse polyclonal claudin-1, occludin and zona occludens-1 (ZO-1) antibodies (diluted 1:400; Abcam Biotechnology) at $4^{\circ} \mathrm{C}$ overnight. After three washes in TBS containing $0.05 \%$ Tween-20, the membranes were reacted with a 1:2000 dilution of alkaline phosphatase-labelled goat anti-rabbit IgG (Jackson Biotechnology) for $2 \mathrm{~h}$ at room temperature. The bound proteins were subsequently detected with the enhanced chemiluminescent detection system (Amersham Biosciences). Quantification of the blots was achieved densitometrically using Quantity One 1-D analysis software (Bio-Rad).

\section{Statistical analysis}

SPSS version 17.0 software (SPSS, Inc.) was used to perform the statistical analyses. The data were expressed as means with their standard errors. Incidences of BT were statically evaluated using the $\chi^{2}$ test. One-way ANOVA was used for multiple comparisons and the least significant difference test was used for intra-group comparison, and $P<0.05$ was considered statistically significant.

\section{Results}

\section{Pretreatment with bifidobacteria modulates intestinal microbiota}

As expected, compared with the sham-operated group, the caecal content colony counts of anaerobes (Lactobacillus, Bacteroides and bifidobacteria) were significantly reduced, while the colony counts of aerobes (Enterobacteria and Enterococcus) were increased in the I/R group at $3 \mathrm{~h}$ after treatment $(P<0 \cdot 01,<0 \cdot 01,<0 \cdot 01,<0 \cdot 05$ and $<0.05$, respectively). After pretreatment with bifidobacteria, counts of anaerobes (Lactobacillus, Bacteroides and bifidobacteria) were significantly increased, while the colony counts of aerobes (Enterobacteria and Enterococcus) were reduced in the I/R group $(P<0 \cdot 01,<0 \cdot 01,<0 \cdot 01,<0.05$ and $<0 \cdot 05$, respectively) (Fig. 1$)$.
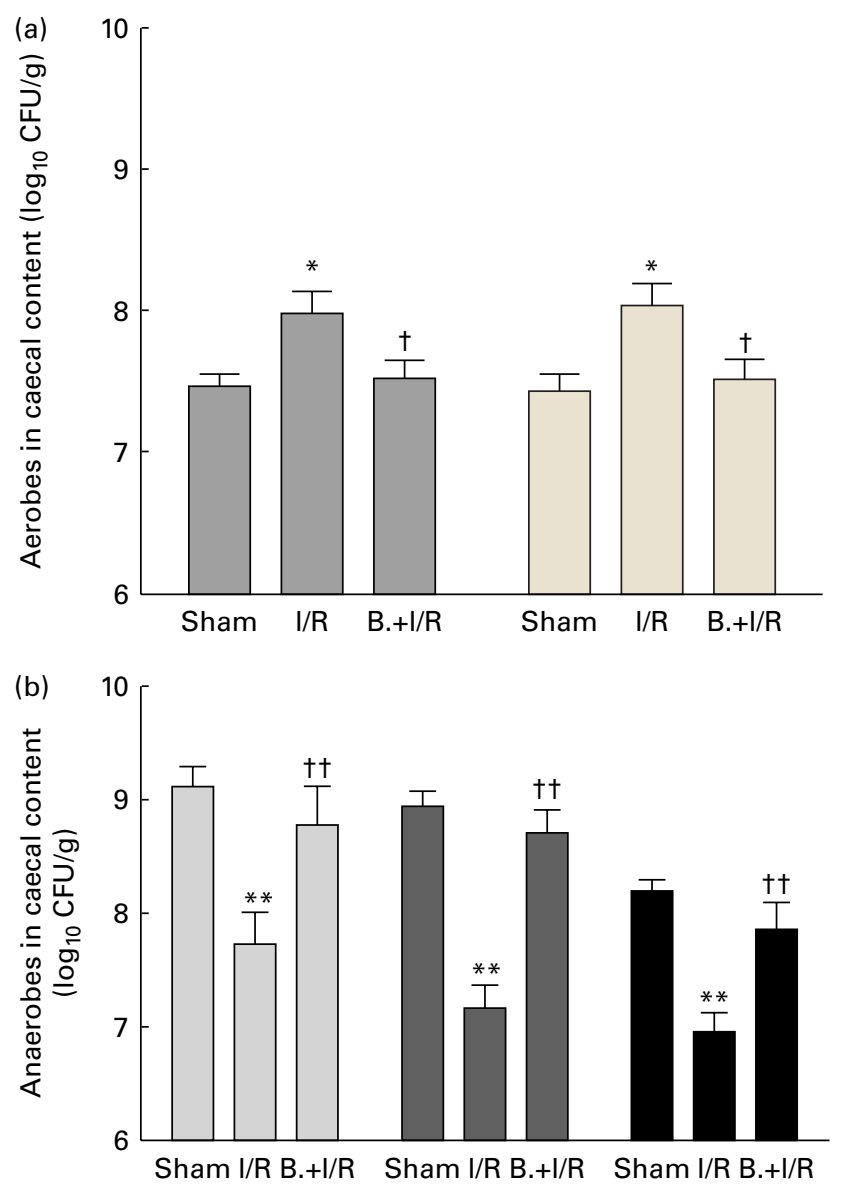

Fig. 1. Caecal content microbiological findings from each group ( $n$ 8). At $3 \mathrm{~h}$ after sham-operated or ischaemia and reperfusion (I/R), the proximal part of the caecum was dissected and caecal content was cultured for colony counts of (a) aerobes ( $\square$, Enterobacteria; $\square$, Enterococcus) and (b) anaerobes ( $\square$, Bacteroides; $\square$, bifidobacteria; $\square$, Lactobacillus). Values are means, with their standard errors represented by vertical bars. Mean values were significantly different compared with that of the sham-operated group: ${ }^{*} P<0.05$, ${ }^{\star *} P<0.01$. Mean values were significantly different compared with that of the I/R group: $\dagger P<0.05$, $\dagger \dagger P<0.01$. The mean colony counts obtained from the agar plates were expressed as colony-forming units (CFU)/g of content. B., mice with bifidobacteria supplement. 
Table 1. Caecal concentrations of SCFA in mice in each group (Mean values with their standard errors)

\begin{tabular}{|c|c|c|c|c|c|c|}
\hline \multirow[b]{3}{*}{ SCFA } & \multicolumn{6}{|c|}{ SCFA contentions $(\mu \mathrm{mol} / \mathrm{g})$} \\
\hline & \multicolumn{2}{|c|}{ Sham } & \multicolumn{2}{|c|}{$\mathrm{I} / \mathrm{R}$} & \multicolumn{2}{|c|}{ B. $+\mathrm{I} / \mathrm{R}$} \\
\hline & Mean & SEM & Mean & SEM & Mean & SEM \\
\hline Total SCFA & 92.67 & 2.56 & $56 \cdot 28^{* *}$ & 1.61 & $109 \cdot 62^{*}+\dagger$ & 3.58 \\
\hline Acetate & 57.08 & 2.32 & $39 \cdot 49^{* *}$ & 0.70 & $64.69^{*}+\dagger$ & 1.21 \\
\hline Propionate & $19 \cdot 88$ & 1.04 & $8 \cdot 71^{\star *}$ & 0.39 & $22.93^{*}+\dagger$ & 0.77 \\
\hline Butyrate & 14.49 & 1.23 & $6 \cdot 93^{\star}$ & 1.06 & $20 \cdot 61+\dagger$ & $2 \cdot 38$ \\
\hline Valerate & 1.22 & 0.14 & 1.15 & 0.07 & 1.39 & 0.09 \\
\hline
\end{tabular}

$\mathrm{I} / \mathrm{R}$, ischaemia and reperfusion; B., mice with bifidobacteria supplement.

Mean values were significantly different compared with that of the sham-operated group: ${ }^{\star} P<0.05,{ }^{* \star} P<0.01$.

Mean values were significantly different compared with that of the $\mathrm{I} / \mathrm{R}$ group: †† $P<0.01$.

\section{Pretreatment with bifidobacteria increased the levels of SCFA}

As shown in Table 1, the total SCFA concentration in the caecal contents of the $\mathrm{I} / \mathrm{R}$ group was significantly less than in the sham-operated group $(P<0 \cdot 01)$. However, pretreatment with bifidobacteria in the $\mathrm{I} / \mathrm{R}$ injury model significantly increased the levels of total SCFA $(P<0 \cdot 01)$. Acetate, propionate and butyrate in the caecum were reduced after $\mathrm{I} / \mathrm{R}$ injury $(P<0.01, \quad<0.01$ and $<0.05$, respectively), while pretreatment with bifidobacteria increased them $(P<0 \cdot 01$, $<0.01$ and $<0.05$, respectively) in the $\mathrm{I} / \mathrm{R}$ injury model. However, the concentration of valerate was not affected.

\section{Pretreatment with bifidobacteria prevents bacterial translocation to distant organs}

In the BT analysis, bacteria were observed only in MLN in the sham-operated group. Induction of intestinal $\mathrm{I} / \mathrm{R}$ resulted in significant BT to the MLN, spleen, liver and kidney $(P<0 \cdot 01$, $\left.\chi^{2}=22 \cdot 44\right)$. However, pretreatment with bifidobacteria significantly reduced BT to MLN and distant organs following $\mathrm{I} / \mathrm{R}$ injury compared with that of the $\mathrm{I} / \mathrm{R}$ mice $(P<0.01$, $\left.\chi^{2}=7.63\right)$. There were also significant differences in BT rates between the sham-operated and bifidobacteria $+\mathrm{I} / \mathrm{R}$ groups $\left(P=0.02, \chi^{2}=5 \cdot 74\right)$ (Table 2$)$.

\section{Pretreatment with bifidobacteria shows lower levels of endotoxin, TNF- $\alpha$ and IL-6}

As shown in Fig. 2, I/R induced a significant $(P<0 \cdot 01)$ increase in plasma endotoxin, TNF- $\alpha$ and IL- 6 in mice compared with the sham-operated mice. However, pretreatment with bifidobacteria showed lower levels of endotoxin, TNF- $\alpha$ and IL- 6 in plasma that were initially induced by $\mathrm{I} / \mathrm{R}$ injury compared with that of $\mathrm{I} / \mathrm{R}$ mice $(P<0.05,<0.01$ and $<0.05$, respectively).

\section{Pretreatment with bifidobacteria alleviates the injury of ileal villi}

As shown in Figs. 3 and 4, intestinal mucosal injury was demonstrated. There was significant haemorrhage, loss of lamina propria villus and gland in the I/R group (Fig. 3(b)). Pretreatment with bifidobacteria (Fig. 3(c)) resulted in moderate villus shedding and injured gland. An apparent atrophy of mucosa and leucocyte infiltration was observed after I/R injury $(P<0 \cdot 01)$. After pretreatment with bifidobacteria, mucosal erosion and leucocyte infiltration were significantly decreased in the I/R group $(P<0 \cdot 01)$.

\section{Pretreatment with bifidobacteria ameliorates the disruption of tight junction morphology}

TJ play an important regulatory role in barrier function. TJ are major determinants in the maintenance of barrier permeability. Transmission electron microscopy analysis was performed to further investigate the impact of $\mathrm{I} / \mathrm{R}$ on the morphology of TJ. As demonstrated in the Fig. 5, in the sham-operated group (Fig. 5(a)), the TJ and desmosomes were intact. In the $\mathrm{I} / \mathrm{R}$ group (Fig. 5(b)), TJ ultrastructure was altered, characterised by decreased electron-dense materials in the $\mathrm{TJ}$ and abnormal desmosomes. In the bifidobacteria $+\mathrm{I} / \mathrm{R}$ group (Fig. $5(\mathrm{c})$ ), more electron-dense materials were present between the adjoining cells near the brush border, which indicated the amelioration of disruption of $\mathrm{TJ}$ morphology.

\section{Pretreatment with bifidobacteria increases the expression of tight junction protein}

Primary components of TJ are transmembrane elements such as occludin and the claudins, as well as peripheral membrane proteins ZO-1. We performed Western blotting analysis to study the effect of $\mathrm{I} / \mathrm{R}$ on the expression of $\mathrm{TJ}$ proteins claudin-1, occludin and ZO-1. In the I/R group, the expression of $\mathrm{TJ}$ proteins claudin-1, occludin and ZO-1 were significantly $(P<0.01)$ decreased compared with the sham-operated group. However, pretreatment with bifidobacteria significantly increased the expression of claudin-1, occludin and ZO-1 $(P<0.01,<0.05$ and $<0.05$, respectively) that were initially reduced by I/R injury (Fig. 6).

\section{Discussion}

The intestine is extremely susceptible to ischaemic injury because it has a high demand on oxygen even in the steady

Table 2. Bacterial translocation to mesenteric lymph nodes (MLN) and distant organs in each treatment group

\begin{tabular}{lccccc}
\hline Group & MLN & Liver & Spleen & Kidney & Translocation $(\mathrm{P} / \mathrm{N})$ \\
\hline Sham & 2 & 0 & 0 & 0 & $2 / 32$ \\
I/R & 7 & 5 & 4 & 4 & $20 / 32^{* *}$ \\
B. + I/R & 4 & 2 & 2 & 1 & $9 / 32^{*}+\dagger$ \\
\hline
\end{tabular}

$\mathrm{P} / \mathrm{N}$, number of positive MLN and distant organs in mice/number of MLN and distant organs tested; I/R, ischaemia and reperfusion; B., mice with bifidobacteria supplement.

Values were significantly different compared with that of the sham-operated group: ${ }^{*} P<0.05$, ${ }^{* *} P<0.01$.

Values were significantly different compared with that of the I/R group: $\dagger \dagger P<0.01$. 


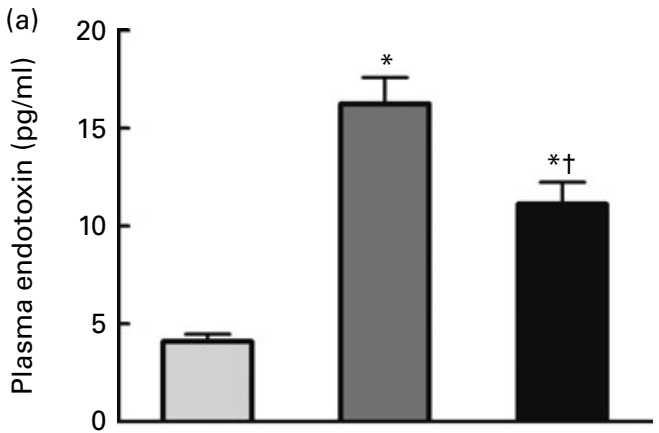

(b)

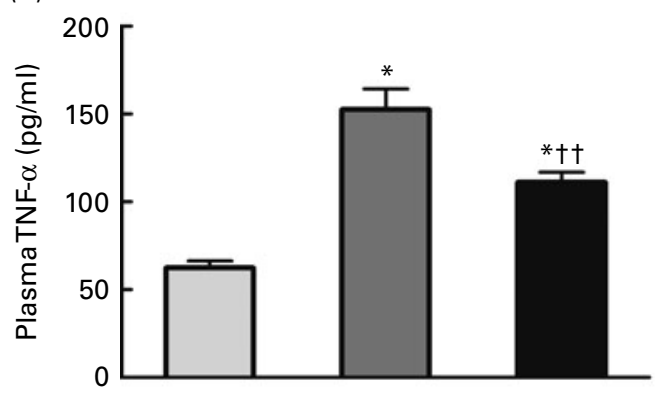

(c)

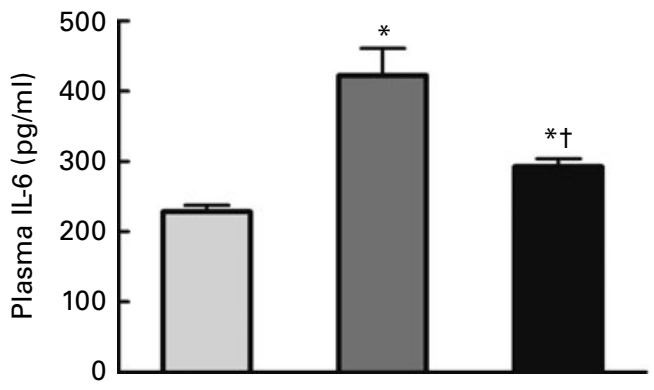

Fig. 2. Levels of (a) endotoxin, (b) TNF- $\alpha$ and (c) IL-6 in the three groups. Values are means, with their standard errors represented by vertical bars. Mean values were significantly different compared with that of the sham-operated group: ${ }^{*} P<0.05$. Mean values were significantly different compared with that of the ischaemia and reperfusion (I/R) group: $\dagger P<0.05$, $\dagger+P<0.01$. B., mice with bifidobacteria supplement. $\square$, Sham; $\square, \mathrm{l} / \mathrm{R} ; \mathbf{\square}, \mathrm{B} .+\mathrm{I} / \mathrm{R}$.

state, and the subsequent reperfusion of the ischaemic intestine deteriorates the ischaemic damage ${ }^{(23,24)}$. Intestinal $\mathrm{I} / \mathrm{R}$ injury has been assumed to be a major factor involved in the process of $\mathrm{BT}$. In the present study, we found that oral pretreatment with bifidobacteria $14 \mathrm{~d}$ before I/R significantly decreased the incidence of bacterial translocation to the MLN and distant organs such as liver, spleen and kidney. Therefore, bifidobacteria used as probiotics seem to have prophylactic protective effects on the gastrointestinal tract. These results are in line with those of Luyer et $a l .{ }^{(25)}$ that pretreatment with Lactobacillus spp. decreases translocation to distant organs. Although the exact mechanism of how bifidobacteria prevent translocation is not known, we can hypothesise that the preventive effect of bifidobacteria appears to modulate gut microbiota. Intestinal potentially pathogenic bacterial overgrowth has been considered an important factor for the development of BT in I/R experimental studies and in patients with acute trauma ${ }^{(26,27)}$. Pathogenic bacteria, which may be present (in very small proportions) as part of the normal microbiota, can become dominant if this equilibrium is disrupted $^{(28,29)}$. Bifidobacteria have been shown to inhibit
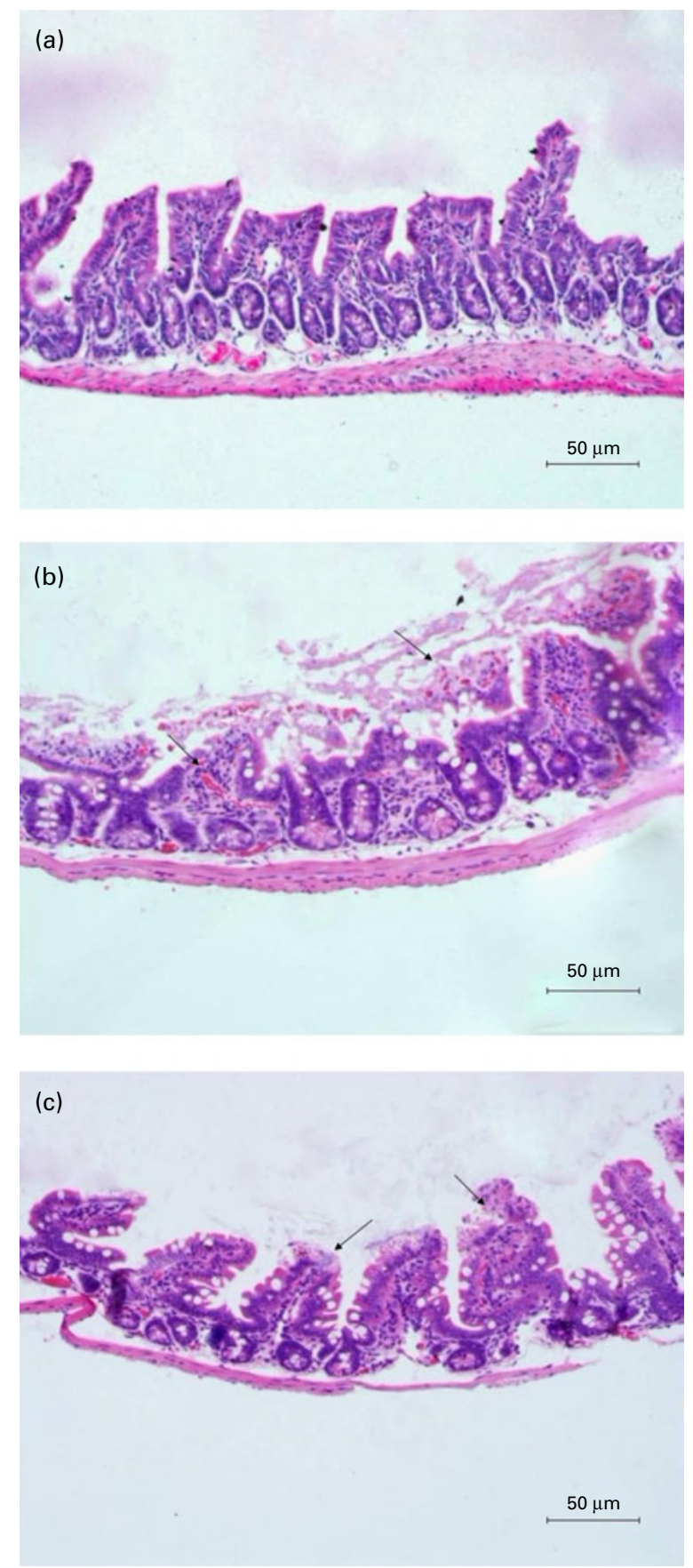

Fig. 3. Histopathology of ileum specimens with representation from each group in the light microscope (haematoxylin and eosin, 100 $\times$ ). (a) Shamoperated group shows normal villous architecture and gland, with no vascular congestion. (b) In ischaemia and reperfusion (I/R) group, intestinal mucosa is severely damaged, as shown by the loss of lamina propria villus and gland and haemorrhage (arrows). (c) In mice with bifidobacteria supplement (B.) + I/R group, pretreatment with bifidobacteria alleviates intestinal I/R injury characterised by moderate villus shedding and injured gland (arrows). Bars $=50 \mu \mathrm{m}$. (A colour version of this figure can be found online at http://journals.cambridge.org/bjn) 


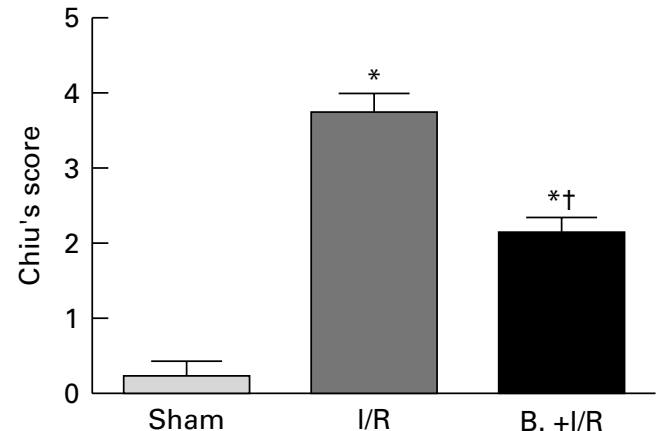

Fig. 4. Histological evaluation (Chiu's score) of intestinal specimens in each treatment group. Values are means, with their standard errors represented by vertical bars. Mean values were significantly different compared with that of the sham-operated group: ${ }^{*} P<0 \cdot 01$. Mean values were significantly different compared with that of the ischaemia and reperfusion (I/R) group: $\dagger P<0.01$. B., mice with bifidobacteria supplement.

the growth of a variety of bacteria and to contribute to the elimination of pathogenic enteric bacteria by producing antimicrobial substances. For instance, Fujiwara et $a l{ }^{(30)}$ described a proteinaceous factor, produced by B. longum SBT 2928, which inhibited adherence of the enterotoxigenic Escherichia coli strain Pb176 to the gangliotetrasylceramide GA1 molecule in vitro, and Fukuda et al. ${ }^{(18)}$ demonstrated that acetate produced by protective bifidobacteria improves intestinal defence mediated by epithelial cells and thereby protects the host against lethal infection. Recent studies have also shown that disruption of the normal intestinal microbiota, resulting in bacterial overgrowth with enteric bacilli, can participate in the production of mesenteric lymph, which is injurious to endothelial cells in a trauma or haemorrhagic-shock model $^{(26)}$, and that a decrease in counts of mucosal bifidobacteria is a significant risk factor for the development of gutderived $\mathrm{BT}$ in thermal-injured rats ${ }^{(31)}$. In the present study, we found that $\mathrm{I} / \mathrm{R}$ injury can lead to a decrease in the number of intestinal obligate anaerobic bacteria, such as Bacteroides, Bifidobacterium and Lactobacillus. Nevertheless, pretreatment with bifidobacteria significantly decreased the counts of aerobic and increased the counts of obligate anaerobic bacteria in the caecal content, and significantly recovered the normal microbiota population after I/R injury. The normal microbiota can prevent colonisation of pathogenic organisms by colonisation resistance, occupying the microenvironment where these organisms could mature steadily. This relationship is highly dependent on the balance of host factors, environmental factors and microbial interactions. These findings suggested an important role of gut microbiota in systemic response after I/R injury. Therefore, the changes in intestinal microbiota observed after $\mathrm{I} / \mathrm{R}$ injury in the present study, at least in part, explain the decrease in BT. The present study has not clarified the mechanism of how the bifidobacteria changed the gut aerobic and anaerobic bacterial concentration. Further investigations about the effects of bifidobacteria on the population of the microbiota in the gut are needed.

SCFA, particularly acetate, propionate and butyrate, are the dominating end products of bacterial fermentation of carbohydrates by intrinsic intestinal digestive enzymes in the large bowel, and are generated by commensal bacteria. These have long been implicated in having a variety of beneficial effects on the host, including trophic and anti-inflammatory effects on the gut in vitro ${ }^{(17,32,33)}$ and in vivo ${ }^{(18,34)}$. In human subjects, SCFA concentration can be measured only in stools, which accounts imperfectly for fermentation
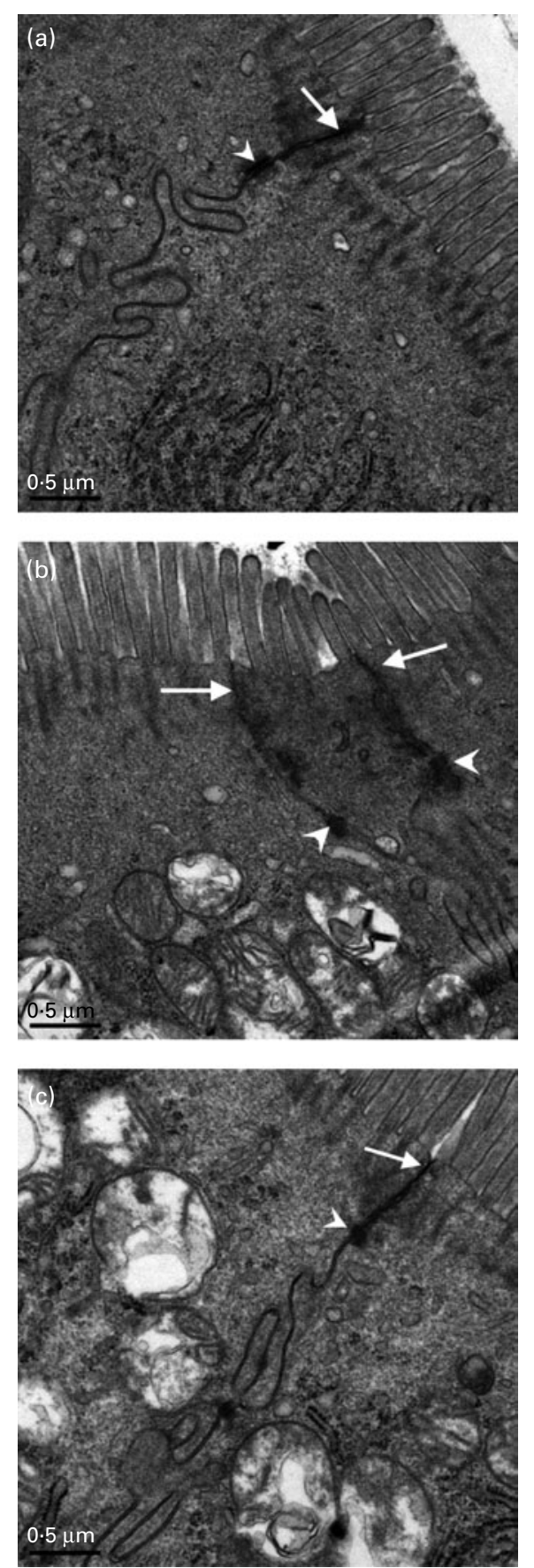

Fig. 5. Transmission electron micrograph of mucosa subjected to ischaemia and reperfusion (I/R) injury. (a) In the sham-operated group, tight junction (TJ) and desmosomes were intact. (b) In the I/R group, TJ ultrastructure was altered, characterised by decreased electron-dense materials in the $\mathrm{TJ}$ and abnormal desmosomes. (c) In mice with bifidobacteria supplement (B.) + I/R group, more electron-dense materials were present between the adjoining cells near the brush border in the I/R injury mice, which indicated the amelioration of disruption of TJ morphology. Arrows, tight junction; arrow heads, desmosome. Bars $=0.5 \mu \mathrm{m}$. 

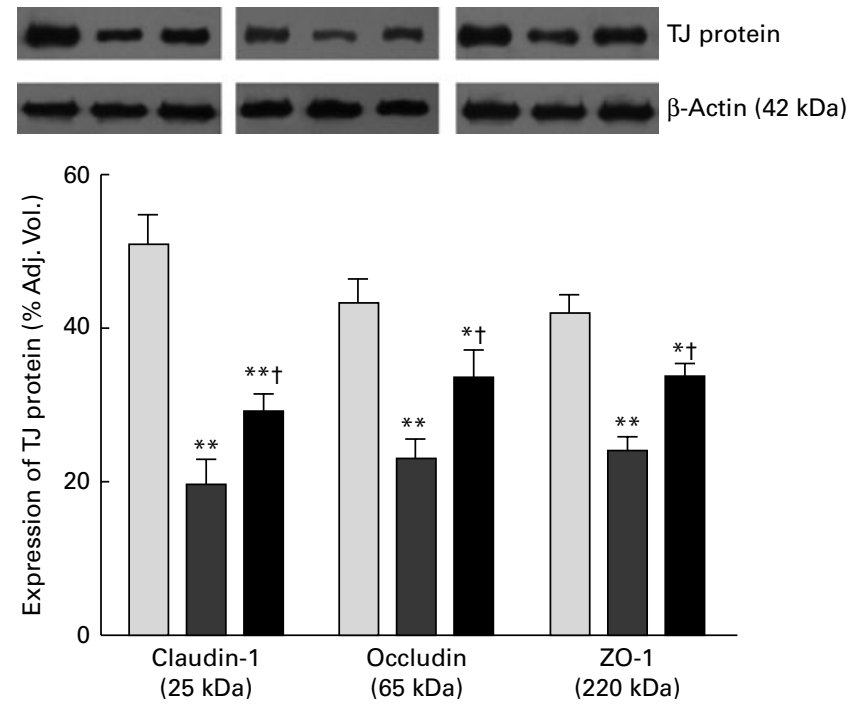

Fig. 6. The expression of tight junction (TJ) proteins claudin-1, occludin and zona occludens-1 (ZO-1) in each group. Values are means, with their standard errors represented by vertical bars. Mean values were significantly different compared with that of the sham-operated group: ${ }^{*} P<0.05$, ${ }^{* *} P<0.01$. Mean values were significantly different compared with that of the ischaemia and reperfusion (I/R) group: $\dagger P<0.05$. B., mice with bifidobacteria supplement; Adj. Vol., adjusted volume. $\square$, Sham; $\square$, I/R; $\mathbf{\square}$, B. + I/R.

occurring mainly in the proximal part of the large intestine ${ }^{(35)}$. Accordingly, we chose to use mice, a model allowing accessibility to caecal contents, which was validated for studies of SCFA ${ }^{(36)}$. The present study demonstrated that the I/R injury significantly decreased the levels of caecal total SCFA, acetate, propionate and butyrate, while pretreatment with bifidobacteria in the $\mathrm{I} / \mathrm{R}$ injury model significantly increased them; these findings are in line with Fukuda et $a l^{(18)}$.

The attachment of the bacteria to the intestinal epithelial cells can elicit the release of cytokines and initiate the subsequent inflammatory response involved in the process of $\mathrm{BT}^{(37)}$. After passing the epithelial barrier, translocating bacteria triggered excessive release of endotoxin and pro-inflammatory factors such as cytokines (TNF- $\alpha$, IL-6, etc.), which have been shown to be important participants in the associated pathophysiology, leading to the exacerbation of intestinal barrier integrity and increased BT during intestinal I/R injury $^{(38,39)}$. In the present study, we found that endotoxin and pro-inflammatory cytokine TNF- $\alpha$ and IL- 6 levels were increased after $3 \mathrm{~h}$ of intestinal $\mathrm{I} / \mathrm{R}$ injury. These results are in accordance with previous studies demonstrating that intestinal I/R plays an important role in cytokine liberation, and treatment with Bifidobacterium infantis and B. bifidum can decrease plasma endotoxin ${ }^{(39,40)}$. Increased TNF- $\alpha$ and endotoxin levels after intestinal $\mathrm{I} / \mathrm{R}$ injury have been associated with increased mortality in animal models ${ }^{(12)}$. The present study confirms the previously documented finding that intestinal $\mathrm{I} / \mathrm{R}$ induces acute pro-inflammatory cytokine and endotoxin release, and promotes the influx of neutrophils into the intestinal mucosa and destroys the intestinal barrier. We found that pretreatment with bifidobacteria showed lower levels of endotoxin, TNF- $\alpha$ and IL- 6 in plasma, which were initially induced by I/R injury. The lower levels of endotoxin,
TNF- $\alpha$ and IL- 6 in plasma, observed in mice pretreated with bifidobacteria, may possibly contribute to reduction of the incidence of BT. These findings suggest that the effects of bifidobacteria on the host immune response cannot be due only to changes in the intestinal microbiota, but may also be due to activation of the monocyte-macrophage system that would increase the capacity of endotoxin clearance from the internal milieu ${ }^{(41-43)}$. Thus, we believe that the beneficial effect of bifidobacteria on intestinal barrier function after $\mathrm{I} / \mathrm{R}$ is mainly mediated through down-regulation of endotoxin and proinflammatory cytokines.

Previous studies have shown that intestinal I/R injury can destroy the intestinal mucosal integrity and contributes to mucosal barrier dysfunction ${ }^{(44)}$. In the present study, the $\mathrm{I} / \mathrm{R}$ injury mice showed higher levels of villous injury in the ileum when compared with the sham-operated mice. However, pretreatment with bifidobacteria can significantly reduce the levels of ileum villous injury. These data confirm that intestinal villous damage occurs in the present experimental model and suggest that it could be related to BT because pretreatment with bifidobacteria decreases ileum mucosal injury and BT, as has been shown in other experimental models of thermal trauma and haemorrhagic shock ${ }^{(7,31)}$. The fact that probiotics protect intestinal mucosal morphological changes in the I/R injury is in line with another study ${ }^{(37)}$ showing that Lactobacillus plantarum L2 can prevent I/R-induced intestinal barrier dysfunction.

$\mathrm{TJ}$ are one of the major determinants of epithelial barrier function. The disruption of TJ can lead to increased paracellular permeability after intestinal ischaemia ${ }^{(45,46)}$. However, the mechanisms by which $\mathrm{I} / \mathrm{R}$ disrupt intestinal barrier function are not fully characterised. In the present study, we found that the morphology of $\mathrm{TJ}$ and the expression of $\mathrm{TJ}$ proteins were changed after $\mathrm{I} / \mathrm{R}$ injury. $\mathrm{I} / \mathrm{R}$ injury led to appearance of aberrant TJ. The disrupted morphology of TJ is often the result of changes in TJ protein expression. Nevertheless, we found that pretreatment with bifidobacteria alleviated $\mathrm{I} / \mathrm{R}$-induced disruption of $\mathrm{TJ}$ in the ileum. These observations showed that I/R-induced changes in TJ structure and function may be associated with the redistribution of $\mathrm{TJ}$ proteins in $\mathrm{TJ}$ membrane microdomains. It denoted the importance of the altered distribution of $\mathrm{TJ}$ proteins in membrane microdomains of $\mathrm{TJ}$ in the preservation of epithelial barrier properties.

\section{Conclusion}

Bifidobacteria may be beneficial in reducing BT in I/R injury of mice. The present data indicate that peroral administration of bifidobacteria is a potential strategy to prevent I/R-induced BT and intestinal barrier dysfunction. However, further studies are required to determine the effects and mechanisms of the relations between certain strains of Bifidobacterium and the host in the present experimental model.

\section{Acknowledgements}

The present work was financially supported by the National Natural Science Foundation of China (grant number 
30830098). The authors declare no conflict of interest. H. W. carried out the majority of the biochemical analysis, designed the experiment and contributed to the writing. W. Z., J. L. and W. Z. contributed to the supervision and drafting of the manuscript. L. Z., B. W. and Q. L. contributed with technical support, scientific advice and revised the manuscript.

\section{References}

1. Sonnenburg JL, Angenent LT, Gordon JI, et al. (2004) Getting a grip on things: how do communities of bacterial symbionts become established in our intestine. Nat Immunol 5, 569-573.

2. Kelly D, Conway S, Aminov R, et al. (2005) Commensal gut bacteria: mechanisms of immune modulation. Trends Immunol 26, 326-333.

3. Hopkins MJ \& Macfarlane GT (2002) Changes in predominant bacterial populations in human faeces with age and with Clostridium difficile infection. J Med Microbiol 51, 448-454.

4. Berg RD \& Owens WE (1979) Inhibition of translocation of viable Escherichia coli from the gastrointestinal tract of mice by bacterial antagonism. Infect Immun 25, 820-827.

5. Wells CL, Vande Westerlo EM, Jechorek RP, et al. (1996) Effect of hypoxia on enterocyte endocytosis of enteric bacteria. Crit Care Med 24, 985-991.

6. Kuzu MA, Kale IT, Col C, et al. (1999) Obstructive jaundice promotes bacterial translocation in humans. Hepatogastroenterology 46, 2159-2164.

7. Ruan X, Shi H, Xia G, Xiao Y, et al. (2007) Encapsulated bifidobacteria reduced bacterial translocation in rats following hemorrhagic shock and resuscitation. Nutrition 23, $754-761$.

8. Peitzman AB, Udekwu AO, Ochoa J, et al. (1991) Bacterial translocation in trauma patients. J Trauma 31, 1083-1086.

9. Farhadi A, Banan A, Fields J, et al. (2003) Intestinal barrier: an interface between health and disease. J Gastroenterol Hepatol 18, 479-497.

10. Sun Z, Wang X, Deng X, Borjesson A, et al. (2000) Phagocytic and intestinal endothelial and epithelial barrier function during the early stage of small intestinal ischemia and reperfusion injury. Shock 13, 209-216.

11. Schoenberg MH, Poch B, Younes M, et al. (1991) Involvement of neutrophils in postischaemic damage to the small intestine. Gut 32, 905-912.

12. Yamamoto S, Tanabe M, Wakabayashi G, et al. (2001) The role of tumor necrosis factor-alpha and interleukin-1beta in ischemia-reperfusion injury of the rat small intestine. J Surg Res 99, 134-141.

13. Ishibashi N \& Yamazaki S (2001) Probiotics and safety. Am J Clin Nutr 73, S465-S470.

14. Westerbeek EA, van den Berg A, Lafeber HN, et al. (2006) The intestinal bacterial colonisation in preterm infants: a review of the literature. Clin Nutr 25, 361-368.

15. Salminen S, Bouley C, Boutron-Ruault MC, et al. (1998) Functional food science and gastrointestinal physiology and function. Br J Nutr 80, Suppl. 1, S147-S171.

16. Harmsen HJ, Wildeboer-Veloo AC, Raangs GC, et al. (2000) Analysis of intestinal flora development in breast-fed and formula-fed infants by using molecular identification and detection methods. J Pediatr Gastroenterol Nutr 30, 61-67.

17. Saulnier DM, Spinler JK, Gibson GR, et al. (2009) Mechanisms of probiosis and prebiosis: considerations for enhanced functional foods. Curr Opin Biotechnol 20, 135-141.
18. Fukuda S, Toh H, Hase K, et al. (2011) Bifidobacteria can protect from enteropathogenic infection through production of acetate. Nature 469, 543-547.

19. Zhang W, Zhu W, Zhang J, et al. (2008) Protective effects of glucagon-like peptide 2 on intestinal ischemia-reperfusion rats. Microsurgery 28, 285-290.

20. Salazar N, Gueimonde M, Hernandez-Barranco AM, et al. (2008) Exopolysaccharides produced by intestinal Bifidobacterium strains act as fermentable substrates for human intestinal bacteria. Appl Environ Microbiol 74, 4737-4745.

21. Chiu CJ, McArdle AH, Brown R, et al. (1970) Intestinal mucosal lesion in low-flow states. I. A morphological, hemodynamic, and metabolic reappraisal. Arch Surg 101, 478-483.

22. Mazzon E \& Cuzzocrea S (2006) Thalidomide treatment reduces the alteration of paracellular barrier function in mice ileum during experimental colitis. Shock 25, 515-521.

23. Dowdall JF, Winter DC, Bouchier-Hayes DJ, et al. (2002) Inosine modulates gut barrier dysfunction and end organ damage in a model of ischemia-reperfusion injury. J Surg Res 108, 61-68.

24. Eror AT, Stojadinovic A, Starnes BW, et al. (1999) Antiinflammatory effects of soluble complement receptor type 1 promote rapid recovery of ischemia/reperfusion injury in rat small intestine. Clin Immunol 90, 266-275.

25. Luyer MD, Buurman WA, Hadfoune M, et al. (2005) Strainspecific effects of probiotics on gut barrier integrity following hemorrhagic shock. Infect Immun 73, 3686-3692.

26. Deitch EA (2002) Bacterial translocation or lymphatic drainage of toxic products from the gut: what is important in human beings. Surgery 131, 241-244.

27. Moore FA, Moore EE, Poggetti RS, et al. (1992) Postinjury shock and early bacteremia. A lethal combination. Arch Surg 127, 893-897, discussion 897-898.

28. Stecher B \& Hardt WD (2001) The role of microbiota in infectious disease. Trends Microbiol 16, 107-114.

29. Dalwai F, Spratt DA, Pratten J, et al. (2006) Modeling shifts in microbial populations associated with health or disease. $A p p l$ Environ Microbiol 72, 3678-3684.

30. Fujiwara S, Hashiba H, Hirota T, et al. (1997) Proteinaceous factor(s) in culture supernatant fluids of bifidobacteria which prevents the binding of enterotoxigenic Escherichia coli to gangliotetraosylceramide. Appl Environ Microbiol $\mathbf{6 3}$, $506-512$

31. Wang Z, Xiao G, Yao Y, et al. (2006) The role of bifidobacteria in gut barrier function after thermal injury in rats. J Trauma 61, 650-657.

32. Tedelind S, Westberg F, Kjerrulf M, et al. (2007) Anti-inflammatory properties of the short-chain fatty acids acetate and propionate: a study with relevance to inflammatory bowel disease. World J Gastroenterol 13, 2826-2832.

33. Kles KA \& Chang EB (2006) Short-chain fatty acids impact on intestinal adaptation, inflammation, carcinoma, and failure. Gastroenterology 130, Suppl. 1, S100-S105.

34. Maslowski KM, Vieira AT, Ng A, et al. (2009) Regulation of inflammatory responses by gut microbiota and chemoattractant receptor GPR43. Nature 461, 1282-1286.

35. Florent C, Flourie B, Leblond A, et al (1985) Influence of chronic lactulose ingestion on the colonic metabolism of lactulose in man (an in vivo study). J Clin Invest 75, 608-613.

36. Pan XD, Chen FQ, Wu TX, et al. (2009) Prebiotic oligosaccharides change the concentrations of short-chain fatty acids and the microbial population of mouse bowel. J Zhejiang Univ Sci B 10, 258-263.

37. Wang B, Huang Q, Zhang W, et al. (2011) Lactobacillus plantarum prevents bacterial translocation in rats following ischemia and reperfusion injury. Dig Dis Sci 56, 3187-3194. 
38. Grotz MR, Deitch EA, Ding J, et al. (1999) Intestinal cytokine response after gut ischemia: role of gut barrier failure. Ann Surg 229, 478-486.

39. Kurtel H, Fujimoto K, Zimmerman BJ, et al. (1991) Ischemia-reperfusion-induced mucosal dysfunction: role of neutrophils. Am J Physiol 261, G490-G496.

40. Griffiths EA, Duffy LC, Schanbacher FL, et al. (2004) In vivo effects of bifidobacteria and lactoferrin on gut endotoxin concentration and mucosal immunity in Balb/c mice. Dig Dis Sci 49, 579-589.

41. Park SY, Ji GE, Ko YT, et al. (1999) Potentiation of hydrogen peroxide, nitric oxide, and cytokine production in RAW 264.7 macrophage cells exposed to human and commercial isolates of Bifidobacterium. Int $J$ Food Microbiol 46, 231-241.
42. Guarner F \& Malagelada JR (2006) Gut flora in health and disease. Lancet 361, 512-519.

43. Zhang LL, Chen X, Zheng PY, et al. (2010) Oral Bifidobacterium modulates intestinal immune inflammation in mice with food allergy. J Gastroenterol Hepatol 25 , 928-934.

44. Hebra A, Hong J, McGowan KL, et al. (1994) Bacterial translocation in mesenteric ischemia-reperfusion injury: is dysfunctional motility the link? J Pediatr Surg 29, 280-285, discussion 285-287.

45. Schoenberg MH \& Beger HG (1993) Reperfusion injury after intestinal ischemia. Crit Care Med 21, 1376-1386.

46. Harhaj NS \& Antonetti DA (2004) Regulation of tight junctions and loss of barrier function in pathophysiology. Int J Biochem Cell Biol 36, 1206-1237. 